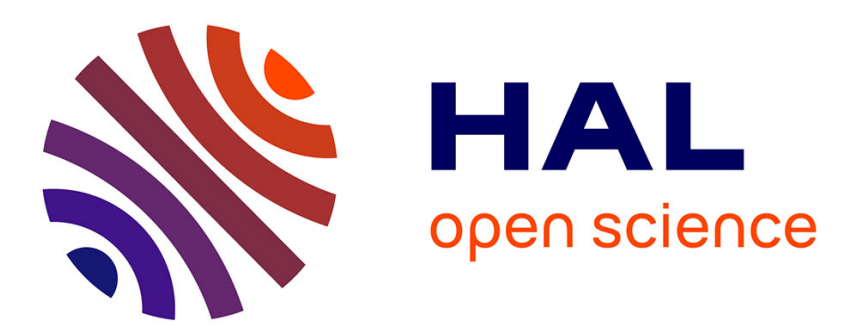

\title{
Ponderomotive Acceleration of Photoelectrons in Surface-Plasmon-Assisted Multiphoton Photoelectric Emission
}

J Kupersztych, P Monchicourt, M Raynaud

\section{- To cite this version:}

J Kupersztych, P Monchicourt, M Raynaud. Ponderomotive Acceleration of Photoelectrons in SurfacePlasmon-Assisted Multiphoton Photoelectric Emission. Physical Review Letters, 2001, 10.1103/PhysRevLett.86.5180 . hal-01327449

HAL Id: hal-01327449

https://hal.science/hal-01327449

Submitted on 8 Jun 2016

HAL is a multi-disciplinary open access archive for the deposit and dissemination of scientific research documents, whether they are published or not. The documents may come from teaching and research institutions in France or abroad, or from public or private research centers.
L'archive ouverte pluridisciplinaire HAL, est destinée au dépôt et à la diffusion de documents scientifiques de niveau recherche, publiés ou non, émanant des établissements d'enseignement et de recherche français ou étrangers, des laboratoires publics ou privés. 


\title{
Ponderomotive Acceleration of Photoelectrons in Surface-Plasmon-Assisted Multiphoton Photoelectric Emission
}

\author{
J. Kupersztych, P. Monchicourt, and M. Raynaud \\ CEA/Saclay, DSM/DRECAM/SPCSI, 91191 Gif-sur-Yvette Cedex, France
}

(Received 27 September 2000)

\begin{abstract}
Photoelectrons emitted from a gold target via a surface-plasmon-assisted multiphoton photoelectric process under a femtosecond laser pulse of moderate intensity are much more energetic than in an ordinary photoeffect without electron collective excitation. The phenomenon is interpreted in terms of time-dependent ponderomotive acceleration of the particles by the resonant field localized at the metal surface. The amplitude of the plasmon resonance may be directly estimated by means of the electron energy spectra.
\end{abstract}

The development of powerful lasers more than three decades ago has allowed the investigation of the generalization of the classical photoelectric emission from metals to processes involving the absorption of several photons [1]. In recent years, the advent of laser pulses of ultrashort duration has favored studies in the femtosecond time regime [2]. These investigations can lead to the creation of new high-current ultrafast electron sources.

Experimental studies have revealed that the electron emission rate can be greatly enhanced by the excitation of collective electron modes of the metal, the so-called surface plasmons $[3,4]$. The increase of the photoelectric signal can be qualitatively explained in terms of an assisted photoelectric effect where the energy of femtosecond light pulses is stored by the surface plasmon, creating a hotelectron population that does not have enough time to transfer its energy to the crystal lattice. While the presence of a surface-plasmon excitation is efficient in increasing the production of photoelectrons, an important open question is how the energy of the emitted electrons in such a "surface-plasmon-assisted" photoelectric process may differ from the energy predicted by the familiar photoelectric equation generalized to multiphoton processes.

In this Letter, we show that the photoelectron energy is strongly affected by the surface-plasmon field, the modification from the classical values depending on the characteristics of the plasmon resonance. This fact may be easily understood by considering a simple analysis of the photoelectron behavior in the inhomogeneous high-frequency electric field surrounding the metal surface. The analysis involves simple classical concepts such as the notion of time-dependent ponderomotive effects, which have been successfully used in the context of multiphoton ionization of atoms in high-intensity lasers [5].

Consider an electron released from the metal surface after having absorbed a required number $n$ of photons from the laser beam to overcome the work function $W$ of the metal. While traveling in the vacuum dressed by the high-frequency field $E_{s p}$ of the surface plasmon, the total energy of the electron consists of the sum of its kinetic energy $\varepsilon_{n}$ (given by the Einstein multiphoton photoelectric equation $\left.\varepsilon_{n}=n \hbar \omega-W\right)$ and its quiver energy $U_{s p}=e^{2} E_{s p}^{2} / 4 m \omega^{2}$ (or ponderomotive potential) in the oscillating electric field. As the field is strongly inhomogeneous, the electron also experiences an effective low-frequency nonlinear force, the ponderomotive force $\vec{F}_{p}=-\vec{\nabla} U_{s p}$; as a result, the particle is expelled from the high-field amplitude regions toward zones of lower field intensity [6]. Consequently, the electron is accelerated toward the vacuum and its quiver energy is converted into kinetic energy. If the electron has enough time to explore the whole spatial extension of the surface plasmon field, which has a finite lifetime $\tau$, the gain of energy can be such that the quiver energy is fully converted ("complete conversion"). Otherwise, only part of the potential energy will be converted ("partial conversion"). As a consequence, if the plasmon lifetime and the laser pulse duration are correlated, the amount of gained kinetic energy depends on the laser pulse duration. In the long pulse regime, where we will show it is possible to have complete conversion of the ponderomotive energy, the minimum extra energy that can be gained by the photoelectrons in a plasmon-assisted photoeffect is simply equal to the ponderomotive potential $U_{s p}$.

In the electron emission in the presence of a surfaceplasmon excitation, another source of energy gain exists that is not, strictly speaking, of ponderomotive origin. A photoelectron may experience an extra acceleration due to the field of the surface plasmon at the instant of its release. This acceleration, which depends on the phase seen by the freed particle [6], will typically last less than half a period of the high-frequency field, and therefore it is not a ponderomotive effect. It can be easily shown [7] that, in the most favorable situation corresponding to an accelerating phase, the maximum kinetic energy that can be transferred to the particle is given by the expression $U_{s p / \max }=3 U_{s p}+2^{3 / 2}\left(\varepsilon_{n} U_{s p}\right)^{1 / 2}$. As a consequence, the photoelectron energy spectrum should show values ranging from $\varepsilon_{\min }=\varepsilon_{n}+U_{s p}$ to $\varepsilon_{\max }=\varepsilon_{n}+U_{s p / \max }$, corresponding to different phases of the plasmon field. 
Hence, a characteristic feature of the photoelectron energy spectrum is that it should exhibit large shifts together with significant broadenings, the order of magnitude of which should correspond to the quantity $2 U_{s p}+2^{3 / 2}\left(\varepsilon_{n} U_{s p}\right)^{1 / 2}$.

The shifting and broadening effects will be less important for shorter laser pulse durations, as the ponderomotive conversion mechanism will be only partial. Thus, the laser pulse length is a fundamental parameter for such timedependent effects. A rough estimate of the pulse durations for which these effects play an important role can be made in the following way. Let us consider, for the sake of simplicity, a photoelectron emitted with a negligible initial velocity that feels a constant ponderomotive force, with a potential of $10 \mathrm{eV}$ (see below). It can be easily shown that the time spent by the particle traveling through the surface-plasmon field of spatial extension $b$ of, let us say, $600 \mathrm{~nm}$ (this value will be justified later on) is, at most, $600 \mathrm{fs}$. Hence, if the plasmon resonance lifetime $\tau$ is longer than this value, the photoelectron will have enough time to cross the whole plasmon field, thus acquiring the kinetic energy corresponding to complete conversion of the ponderomotive potential into kinetic energy. If instead $\tau$ is much shorter than $600 \mathrm{fs}$, the corresponding energy gain will be smaller. As a consequence, effects due to the plasmon lifetime, that we will show are roughly of the same order of the laser pulse duration, will be best manifested by using ultrashort laser pulses in the range of a few hundreds of femtoseconds. It is worth noting that, for much longer laser pulse durations, the ponderomotive energy shifts and broadenings can be smaller because of other relaxation mechanisms such as plasmon-phonon interactions that will take place on longer time scales [8]. These other mechanisms will contribute to decrease the amplitude of the surface-plasmon resonance, thereby lowering the ponderomotive potential and decreasing the energy shifts.

The previously discussed energy shifts and broadenings are significant enough to be effectively measured, as we have shown in our experiment. We have used a gold metal target, the work function of which is $W=5.1 \mathrm{eV}$ [9], and a laser of wavelength $\lambda_{0}=800 \mathrm{~nm}$. As the photon energy $\left(\hbar \omega_{0}=1.55 \mathrm{eV}\right)$ is below the threshold $(2.3 \mathrm{eV})$ for interband transitions [10], plasmon excitation is not expected to be strongly damped. The photoeffect we study is actually a four-photon process. For such a nonlinear process to yield significant results, rather high laser intensities, typically of the order of $10^{9} \mathrm{~W} / \mathrm{cm}^{2}$, are required. If we assume the ratio $\eta=E_{s p} / E_{L}$ between the resonant plasmon amplitude $E_{s p}$ and the amplitude $E_{L}$ of the driving laser field to be of the order of $\eta \cong 200$, the order of magnitude of the energy shifts, characterized by the quantity $U_{s p}=$ $\eta^{2} U_{0}$, where $U_{0}($ in $\mathrm{eV})=9.33 \times 10^{-20} \lambda_{0}^{2} I_{L}$ (with the laser intensity $I_{L}$ in W/ $\mathrm{cm}^{2}$ and the wavelength $\lambda_{0}$ in $\mathrm{nm}$ ), will be at least $2.4 \mathrm{eV}$, a value which may be easily experimentally detected. The time dependence of the shifts and broadenings can be experimentally verified by making the plasmon lifetime (which is the relevant physical parameter) to vary. However, as the experimental parameter is actually the laser pulse duration, an experimental analysis of the relationship between the plasmon lifetime and the laser pulse duration is required in advance.

It has been known since the beginning of the century that light may excite surface-plasmon modes on gold surfaces if these are modulated to form diffraction gratings [11]. Periodic modulation of metallic surfaces provides the extra momentum necessary to satisfy momentum conservation in photon-plasmon scattering. If $\theta$ is the angle of incidence of the laser and $a$ denotes the grating parameter, the momentum $k_{0}$ of the laser photons of angular frequency $\omega_{0}$ traveling along the surface is given by the relation $k_{0}=\left(\omega_{0} / c\right) \sin \theta+2 \pi a^{-1}$ (for the first harmonic) [11], while the surface-plasmon momentum $k$ is related to its frequency $\omega$ by the well-known dispersion relation (in the simplest dielectric approach): $k=(\omega / c)[\varepsilon /(1+\varepsilon)]^{1 / 2}$, where $\varepsilon(\omega, k)$ is the dielectric function of the metal. A simple estimate of the relevant resonance angle $\theta$ (defined by $\omega=\omega_{0}$ and $k=k_{0}$ ) is readily obtained by taking the tabulated experimental value $(\varepsilon \cong-22.3$ at laser wavelength $\lambda_{0}=800 \mathrm{~nm}$ ) [11], which gives, under the conditions of the experiment described hereafter, for grating constant $a=6.67 \mu \mathrm{m}$ corresponding to 150 grooves per millimeter, a value of $\theta \cong 64.6^{\circ}$.

The experiment has been carried out by using a Ti:sapphire laser capable of delivering $85 \mathrm{~mJ}$ laser pulses at $\lambda_{0}=800 \mathrm{~nm}$ with pulse duration adjustable from 60 to $800 \mathrm{fs}$. We used a commercial, sinusoidal, holographically registered grating, covered by $200 \mathrm{~nm}$-thick vacuum-deposited $\mathrm{Au}$ film, held in a magnetically shielded, high vacuum $\left(10^{-8} \mathrm{mbar}\right)$ chamber. The laser beam, the incidence plane of which is perpendicular to the grating grooves, is impinging onto the target without focalization. The laser intensity is deduced from a calorimetric measurement of the pulse energy (with shot-to-shot fluctuations of 5\%) and from the autocorrelation determination of the pulse duration (with a $10 \%$ uncertainty). Electron emission has been analyzed with a time-of-flight, magnetically shielded, spectrometer (of length $60 \mathrm{~cm}$ ), with energy resolution of $0.1 \mathrm{eV}$. All the data have been obtained at maximum of resonance (that is, maximum number of emitted electrons) at an experimental incidence angle $\theta \cong 64.8^{\circ}$, in agreement with the above estimated value. The experimentally determined plasmon wave vector is then $k \cong 8 \times 10^{4} \mathrm{~cm}^{-1}$, which leads to a value of the order of $b \cong 640 \mathrm{~nm}$ for the plasmon field extension in vacuum, as it follows from the theoretical expression: $\quad b=\left(k^{2}-\omega^{2} / c^{2}\right)^{-1 / 2}$. This estimate confirms that effects due to the plasmon lifetime will be best manifested by using ultrashort laser pulses in the range of a few hundreds of femtoseconds.

The measurements of the plasmon lifetime have been performed using an autocorrelation technique: The laser beam is split through a Michelson interferometer into two 
pulses of equal intensities $I_{L}$, with a relative time delay that can be adjusted with an accuracy better than $1 \mathrm{fs}$. As each pulse yields its own photoeffect in the presence of its own plasmon resonance, electron emission, which is a four-photon process, will scale as $2 \times\left(I_{L}\right)^{4}$ if the delay between the two pulses is large, and $\left(2 \times I_{L}\right)^{4}$ if the delay is zero. The variations of the electron signal between these two amplitudes are therefore expected to occur for time delays of the order of the plasmon lifetime. The results of this "pump-pump" technique are shown on Fig. 1. The observed contrast ratio between zero and large delays is close to 8 , as expected. The autocorrelation times (time delay at half maximum) are $80 \mathrm{fs}$ for laser pulses of $60 \mathrm{fs}$, and about $400 \mathrm{fs}$ for pulse durations of $400 \mathrm{fs}$. Thus, in both cases, the overlap between the two plasmon resonances occurs during a period of time close to the laser pulse duration. One may therefore conclude that the plasmon lifetime is of the same order as the laser pulse duration, and this result validates the above analysis.

Investigations on electron emission as a function of the laser intensity have then been performed. The datasets, reported as Lux-Ampère characteristics as in [4], exhibit a slope of 4, as expected from a four-photon photoelectric effect. Our measurements do not show appreciable space charge fields for laser intensities lower than, roughly, $10^{10} \mathrm{~W} / \mathrm{cm}^{2}$. The expected strong dependence of electron emission on the laser polarization $[3,11]$ has been verified: An enhancement of the electron signal by a factor close to 100 has been measured between $s$ and $p$ polarizations. The spectra obtained for a $p$-polarized laser pulse of $60 \mathrm{fs}$ are reported in Fig. 2 as a function of the laser intensity. At the lowest intensity $\left(1.6 \times 10^{9} \mathrm{~W} / \mathrm{cm}^{2}\right)$, the signal corresponds to electron energies ranging from 0 to $1 \mathrm{eV}$ in agreement with the expected value $(1.1 \mathrm{eV})$, which can be deduced from the Einstein multiphoton photoelectric law. As the laser intensity increases, the maximum of energy distribution is seen to be progressively shifted toward

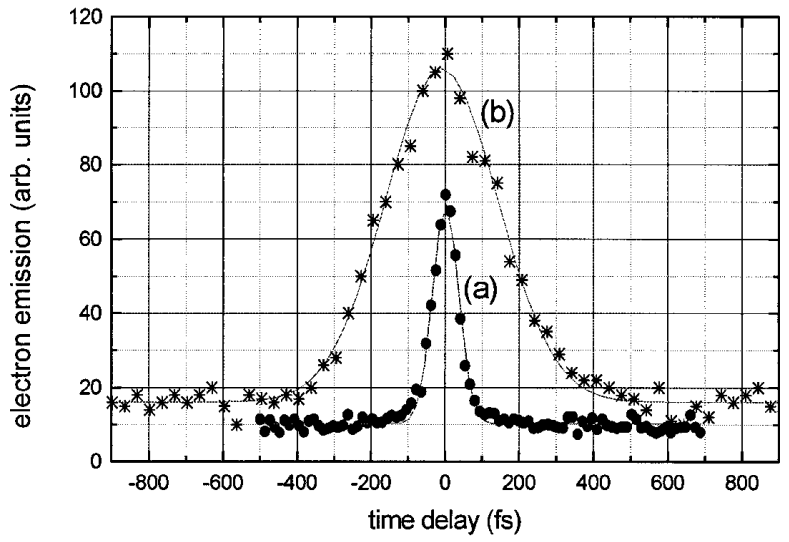

FIG. 1. Variation of the electron signal emitted by an Au surface in the presence of a surface plasmon resonance, irradiated by two laser pulses of equal intensities, as a function of their relative delay for two pulse durations: $60 \mathrm{fs}$ (a); $400 \mathrm{fs}$ (b). much higher energies and the spectrum is broadened $\left(13 \mathrm{eV}\right.$ at $\left.8 \times 10^{9} \mathrm{~W} / \mathrm{cm}^{2}\right)$. However, conversion of the ponderomotive potential into electron kinetic energy has not time to be fully achieved for this ultrashort pulse duration (60 fs), thus, larger shifts may be expected when the laser pulse duration, that is, the plasmon lifetime, is increased. Such a behavior has been experimentally verified, as shown on Fig. 3. For a fixed laser intensity $\left(I_{L}=3.2 \times 10^{9} \mathrm{~W} / \mathrm{cm}^{2}\right)$, the top of the electron distribution is shifted by ca. $2.5 \mathrm{eV}$ for a pulse duration of $60 \mathrm{fs}$, and by $16 \mathrm{eV}$ for $190 \mathrm{fs}$. The shifts evolve then more slowly: $18 \mathrm{eV}$ for $400 \mathrm{fs}$ and $25 \mathrm{eV}$ for $800 \mathrm{fs}$. According to this evolution, our data are consistent with a nearly complete conversion of the ponderomotive potential into kinetic energy during the longest pulse duration, i.e., 800 fs. Thus, photoelectrons with kinetic energies up to $40 \mathrm{eV}$ have been produced in a plasmon-assisted photoeffect with moderate laser intensities, whereas electron energies of less than $1.1 \mathrm{eV}$ are expected from the ordinary multiphoton photoelectric process. Another significant feature is the fairly Gaussian shape of the distributions with a FWHM of the order of the shifts. As already mentioned, the physical origin of these large broadenings is the initial acceleration that depends on the random phase "seen" by the electrons when they are released in the plasmon field. Numerical simulations show that the observed shape of the spectra effectively results from a nonadiabatic (nonponderomotive) acceleration mechanism of the photoelectron population at the very beginning of the interaction [12].

An interesting result that can be obtained from the photoelectron energy spectra and the simple model developed here is the possibility of evaluating the amplitude of the plasmon resonance. Thus, if we consider the case of a $800 \mathrm{fs}$ pulse duration which corresponds to full conversion

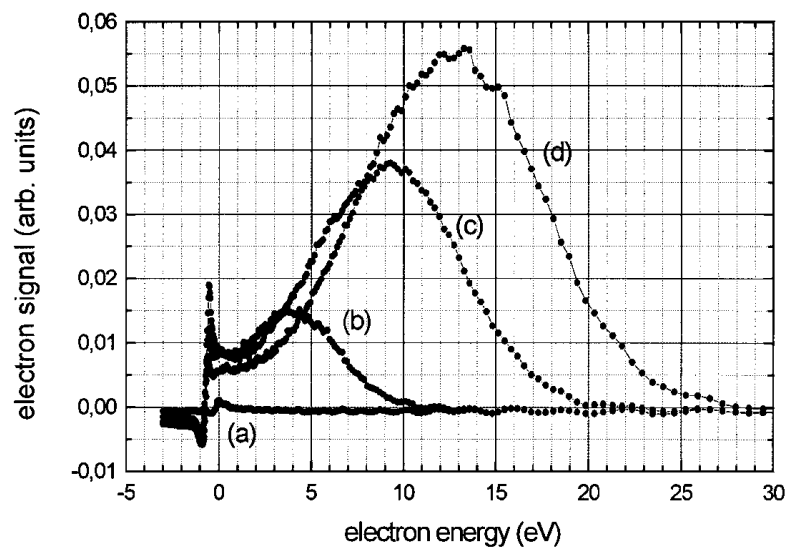

FIG. 2. Photoemitted electron spectra (raw data) of the Au surface irradiated with a 60 fs laser pulse for four laser intensities: $1.6 \times 10^{9} \mathrm{~W} / \mathrm{cm}^{2}$ (a), $4 \times 10^{9} \mathrm{~W} / \mathrm{cm}^{2}$ (b), $6.5 \times 10^{9} \mathrm{~W} /$ $\mathrm{cm}^{2}$ (c), and $8 \times 10^{9} \mathrm{~W} / \mathrm{cm}^{2}$ (d). The overshoot near zero energy is due to transient effects in the spectrometer and concerns a negligible part of the electron population. 


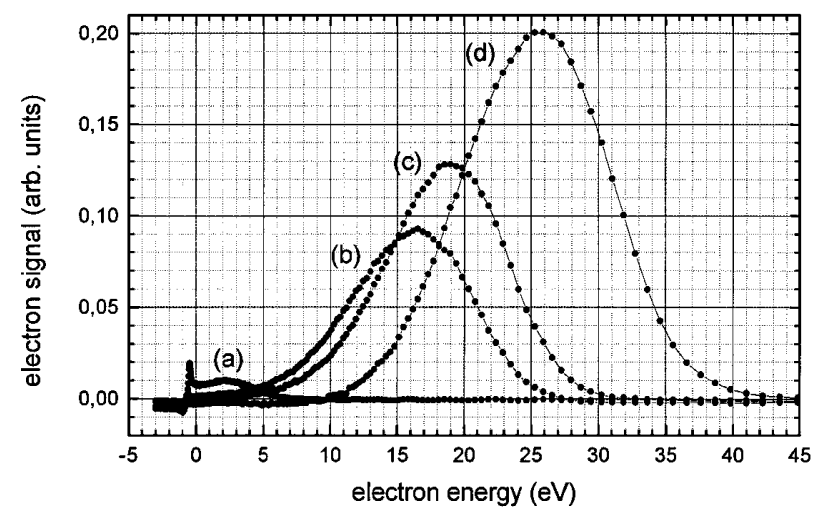

FIG. 3. Photoemitted electron spectra (raw data) at fixed laser intensity $\left(3.2 \times 10^{9} \mathrm{~W} / \mathrm{cm}^{2}\right)$ and for four pulse durations: $60 \mathrm{fs}(\mathrm{a}), 190 \mathrm{fs}(\mathrm{b}), 400 \mathrm{fs}(\mathrm{c})$, and $800 \mathrm{fs}(\mathrm{d})$.

of the electron quiver energy into kinetic energy, it appears that the corresponding spectrum presents a broadening of about $30 \mathrm{eV}$ together with a minimum electron energy of nearly $\varepsilon_{\min } \cong 11 \mathrm{eV}$. Hence, the ponderomotive energy is about $U_{s p} \cong 10 \mathrm{eV}$ and the theoretical value of the broadening, given by the expression $2 U_{s p}+2^{3 / 2}\left(\varepsilon_{n} U_{s p}\right)^{1 / 2}$, is about $29 \mathrm{eV}$, for $\varepsilon_{n}=1.1 \mathrm{eV}$, in excellent agreement with the experimental measurement. From the value of the laser intensity $\left(I_{L}=3.2 \times 10^{9} \mathrm{~W} / \mathrm{cm}^{2}\right)$, we find $U_{0} \cong 1.9 \times 10^{-4} \mathrm{eV}$ and the deduced amplitude ratio $\eta$ is therefore of the order of 230 . It should be pointed out that previous experiments on reflectivity or transmission by thin films using spectrophotometry and polarimetry techniques involving continuous light sources [13] have led to estimate significantly lower values for the plasmon resonance [11]. However, these experiments have involved steady-state irradiation conditions with electrons in equilibrium with the lattice, which is obviously not the case in the ultrashort time conditions of the present experiments (60 to $800 \mathrm{fs}$ ), where the main relaxation mechanisms such as plasmon-phonon interactions [8] do not have enough time to significantly affect the plasmon resonance amplitude.

In summary, we have shown that the photoelectrons emitted by a metallic surface with a surface-plasmonassisted photoeffect are much more energetic than in an ordinary photoeffect without electron collective excitation. Moreover, there is no need of very high laser intensities, such as in the case of multiphoton ionization of atoms [5], to obtain efficient ponderomotive accelerations as the relevant strong electric field is not the laser wave but the resonant field of the plasmon localized at the metal surface. An interesting consequence of this analysis is that the electron spectra provide a way to evaluate the magnitude of the surface-plasmon resonance.

The authors thank S. Bastiani, F. Coat, A. DeSmedt, and M. Lesage for their participation in various experiments, together with the technical assistance of the Saclay laser facility.

[1] See, e.g., E. M. Logothetis and P.L. Hartman, Phys. Rev. 187, 460 (1969); J.H. Bechtel, W. Lee Smith, and N. Bloembergen, Phys. Rev. 15, 4557 (1977); R. Yen, J. Liu, and N. Bloembergen, Opt. Commun. 35, 277 (1980).

[2] J. G. Fujimoto, J. M. Liu, E. P. Ippen, and N. Bloembergen, Phys. Rev. Lett. 53, 1837 (1984); G. Ferrini, A. Viggiani, D. Sertore, P. Michelato, and F. Parmigiani, Phys. Rev. B 60, 8383 (1999).

[3] J. G. Endriz and W. E. Spicer, Phys. Rev. 24, 64 (1970); T. Tsang, T. Srinivasan-Rao, and J. Fischer, Opt. Lett. 15, 866 (1990); T. Stuckless and M. Moskovits, Phys. Rev. B 40, 9997 (1989); V. M. Shalaev, C. Douketis, T. Haslett, T. Stuckless, and M. Moskovits, Phys. Rev. B 53, 11193 (1996).

[4] P. Monchicourt, M. Raynaud, H. Saringar, and J. Kupersztych, J. Phys. Condens. Matter 9, 5765 (1997).

[5] P. Agostini, J. Kupersztych, L. A. Lompré, G. Petite, and F. Yergeau, Phys. Rev. A 36, 4111 (1987); R. R. Freeman, P. H. Bucksbaum, H. Milchberg, S. Darack, D. Schumacher, and M.E. Geusic, Phys. Rev. Lett. 59, 1092 (1987); J. Kupersztych, L. A. Lompré, G. Mainfray, and C. Manus, J. Phys. B, At. Mol. Opt. Phys. 21, L517 (1988); J. Kupersztych, Phys. Scr. 42, 51 (1990).

[6] J. Kupersztych, Phys. Rev. Lett. 54, 1385 (1985).

[7] J. Kupersztych and M. Raynaud (to be published).

[8] H. E. Elsayed-Ali, T. B. Norris, M. A. Pessot, and G. A. Mourou, Phys. Rev. Lett. 58, 1212 (1987); R. W. Schoenlein, W.Z. Lin, and J. G. Fujimoto, Phys. Rev. Lett. 58, 1680 (1987).

[9] A. H. Sommer, Photoemissive Materials (Krieger, Huntington, 1980).

[10] B. R. Cooper, H. Ehrenreich, and H. R. Philipp, Phys. Rev. 138, 494 (1965).

[11] See, e.g., H. Raether, Surface Plasmons (Springer-Verlag, Berlin, 1988).

[12] C. Riconda, J. Kupersztych, P. Monchicourt, and M. Raynaud (to be published).

[13] P. B. Johnson and R. W. Christy, Phys. Rev. B 6, 4370 (1972); U. Schröder, Surf. Sci. 102, 118 (1981). 